\title{
Infecção de corrente sanguínea relacionada a cateter venoso central (ICSRC) em enfermarias: estudo prospectivo comparativo entre veia subclávia e veia jugular interna
}

\author{
Catheter-associated bloodstream infections (CA-BSI) in wards: a prospective comparative \\ study between subclavian and jugular access
}

Gustavo Lopes Gomes Siqueira', Walkiria Hueb², Rodrigo Contreira 3 , Maria Aparecida Nogueron4, Daniela Muniz Cancio4, Roberto Augusto Caffaro 5

\begin{abstract}
Resumo
Contexto: Hemocultura positiva associada a cateter venoso central tem sido estudada em unidades de terapia intensiva (UTI), mas ainda é controverso se o acesso jugular tem maior incidência de complicações infecciosas que o acesso na veia subclávia.

Objetivo: Comparar índice de infecção entre os acessos na jugular interna e os na veia subclávia em pacientes internados nas enfermarias de cirurgia. Métodos: Estudo prospectivo, descritivo e comparativo com 114 cateteres em 96 pacientes admitidos nas enfermarias de cirurgia de um Hospital Quaternário, tendo como variáveis o local de inserção, número de lumens, tempo de uso, comparando-os com o índice de complicações infecciosas. Resultados: $O$ índice de infecção foi de 9,64\% (11 cateteres), sem significância estatística quando comparados o número de lumens (mono versus duplo) e infecção ( $p=0,274)$; também sem significância estatística a comparação entre o tempo de uso ( $\geq 14$ dias) e infecção ( $p=0,156)$. Comparando os acessos jugular e subclávia, encontramos significância estatística tendo infecção em 17,2\% na subclávia e 1,8\% na jugular, com p=0,005. Índice de Hemocultura positivo associado a cateter venoso central foi maior no acesso subclávia quando comparado com jugular interna, com OR 11,2, IC95\% $(1,4-90,9 ; p=0,023)$.

Conclusões: $\mathrm{O}$ acesso venoso central na jugular interna tem menor risco de infecção se comparado com subclávia em enfermarias.
\end{abstract}

Palavras-chave: cateterismo venoso central; infecção hospitalar; bacteriemia.

\begin{abstract}
Background: Positive hemoculture associated with central venous catheters has been studied in intensive care units (ICU), but is still controversial if the internal jugular vein access has a higher incidence of infection than subclavian or femoral vein access.

Objective: To compare catheter-related bloodstream infection (CABSI) rates between internal jugular and subclavian vein access in patients admitted to surgical wards.

Methods: This is a prospective, descriptive and comparative study of 114 central venous catheters placed in 96 patients admitted to the surgical wards of a tertiary-care hospital. The following parameters were studied: local of insertion of the catheter (internal jugular versus subclavian), number of lumens (single versus double) and duration of use (longer or shorter than 14 days), in order to determine their influence in CABSI rates.

Results: The CABSI rate was 9,64\% (11 catheters), with no significant statistical differences regarding the number of lumens ( $p=0.274$ ), and duration of use $(p=0.156)$. The CABSI rate was higher in the subclavian vein than in the internal jugular vein access (OR 11.2, 95\% Cl 1.4-90.8; $p=0.023$ ).

Conclusions: The internal jugular vein access has a lesser incidence of CABSI than subclavian vein access in patients admitted to surgical wards.
\end{abstract}

Keywords: catheterization, central venous; cross infection; bacteremia.

Trabalho realizado na Irmandade Santa Casa de Misericórdia de São Paulo - São Paulo (SP), Brasil.

'Médico do setor de Cirurgia Vascular e Endovascular do Hospital João XXIII - Campina Grande (PB), Brasil. Ex-residente do Departamento de Cirurgia Vascular da Santa Casa de São Paulo -

São Paulo (SP), Brasil. Membro da Sociedade Brasileira de Cirurgia Vascular.

${ }^{2}$ Médica do Departamento de Cirurgia Vascular; chefe do Grupo de Acessos Vasculares (GAV) da Santa Casa de São Paulo - São Paulo (SP), Brasil. Membro do Vascular Access Society.

${ }^{3}$ Médico assistente do Setor de Infectologia da Santa Casa de São Paulo - São Paulo (SP), Brasil.

${ }^{4}$ Enfermeira do Grupo de Acessos Venosos (GAV) da Santa Casa de São Paulo - São Paulo (SP), Brasil.

${ }^{5}$ Médico-chefe do Departamento de Cirurgia Vascular da Santa Casa de São Paulo - São Paulo (SP), Brasil.

Conflito de interesses: nada a declarar.

Submetido em: 01.07.10. Aceito em: 03.06.11. 


\section{Introdução}

O aparecimento de múltiplas técnicas e desenvolvimento tecnológico relacionado ao acesso vascular permitiu o salvamento, assim como o prolongamento, da vida de incontáveis pacientes. Mas sua utilização não está isenta de complicações, pois muitas vezes podem evoluir de forma catastrófica. Assim, se faz necessária a devida atenção desde a inserção até a sua retirada.

Estima-se que só nos Estados Unidos (EUA) cinco milhões de cateteres venosos centrais são inseridos por ano. A infecção de corrente sanguínea relacionada a estes ocupa o terceiro lugar como causa de infecção hospitalar $(14 \%)^{1}$, como conseguinte tem-se o prolongamento do tempo de internação de 7 a 19 dias, com taxa de mortalidade, que vem alcançando picos de $25 \% \%^{2,3}$.

São fatores de risco para esta complicação: a) duração em dias do uso do cateter; b) tipo do cateter; c) número de lumens; d) tipo de infusão; e) técnica de inserção; f) sítio de inserção ${ }^{4,5}$. Está bem estabelecido que a taxa de infecção em relação ao sítio de inserção do cateter venoso central (CVC) é maior quando se opta pela via inguinal, em comparação com a via jugular interna e subclávia. No entanto, na literatura não há consenso quando comparadas as últimas vias entre si. Observa-se uma tendência nos trabalhos para maior taxa de infecção quando o sítio é jugular, porém, esta realidade compreende apenas os estudos em pacientes de UTI ${ }^{5,6}$.

São escassos os estudos na literatura que foram realizados em ambientes externos à UTI. Marschall et $\mathrm{al}^{7}$, narram que ao efetuarem uma busca no site da National Library Medicine, em 2007, com os termos bloodstream, infecction e non-ICU, obtiveram como resultado somente quatro trabalhos na literatura. O primeiro grande estudo observacional, de caráter meramente descritivo, foi realizado na Alemanha e publicado em 2006; nele foi encontrado alto índice de infecção, medindo 4,3/1000 cateteres por dia, marcadamente mais alto que $1,8 / 1000$ cateteres por dia em $\mathrm{UTI}^{8}$.

Nos EUA, o primeiro grande estudo observacional foi publicado em 2007 e apresentou resultado semelhante ao descrito acima, no que tange a comparação da infecção de cateter em UTI ${ }^{7}$. Tal fato gerou controvérsias, vez que se esperava que em ambientes externos à UTI, com pacientes de menor gravidade, o índice de infecção fosse menor. Estudo transversal, publicado em 2004 por Trick et al. ${ }^{9}$ evidenciou que $83 \%$ dos acessos venosos passados sem justificativa foram em enfermarias, sendo em porcentagem $1,8 \%$ dos acessos em UTI contra 8,5\% dos acessos em enfermarias, sugerindo que um dos fatores predisponentes de infecção em enfermaria fosse a maior quantidade de acessos clinicamente injustificáveis.

Não existem estudos realizados no Brasil sobre tal assunto, nem na literatura mundial, comparando os diferentes locais de inserção com relação à infecção.

Assim, devido à frequência da instalação de CVC em pacientes cirúrgicos, às complicações graves relacionadas aos mesmos, ao impacto econômico que uma bacteremia ou até septicemia determinam ao tratamento de cada paciente, e à ausência de estudos na literatura relacionados às enfermarias, sentimo-nos motivados a esclarecer qual das vias de acesso apresentam maior taxa de infecção na corrente sanguínea relacionada ao cateter (ICSRC).

\section{Casuística e método}

No período compreendido entre 11 de março a 11 de junho de 2009, iniciou-se um estudo prospectivo, descritivo e comparativo nas Enfermarias das Unidades de Cirurgia do Hospital Central da Santa Casa de São Paulo, onde 96 pacientes tiveram 114 cateteres acompanhados através de preenchimento de protocolo, desde sua colocação até consequente retirada.

Critérios de inclusão: todos os pacientes que necessitavam de acesso venoso central, puncionados em veia jugular interna ou subclávia, que estavam em enfermarias e tinham o protocolo específico preenchido.

Critérios de exclusão: não preenchimento adequado do protocolo e punção de qualquer outro acesso central que não em subclávia ou jugular interna.

A passagem do cateter foi realizada por médicos residentes da anestesiologia ou cirurgia, em ambiente cirúrgico ou nas enfermarias das Unidades de Cirurgia, sempre através de técnica asséptica. Não houve discriminação em relação ao ambiente em que foi realizada a cateterização do acesso ao nosso protocolo. A escolha do sítio de punção entre jugular e subclávia foi aleatório e não foi computado se houve conversão de um sítio para outro durante o procedimento.

Foram coletadas informações referentes à evolução de cada cateter, conforme protocolo previamente estabelecido, por duas enfermeiras especializadas designadas pelo Grupo de Acesso Vascular da Santa Casa de São Paulo (GAV).

Critérios para ICSRC: hemoculturas pareadas do sangue periférico e CVC positivas apresentando tempo de positividade mais rápido para o sangue proveniente do cateter, assim como maior quantidade do agente infeccioso no dispositivo e crescimento do mesmo germe nas amostras. Isto associado a sinais clínicos de infecção, como febre e/ou 
calafrios, sendo descartada infecção da corrente sanguínea de outra natureza. Nossos critérios foram determinados pelo Guideline do CDC de $2002^{6}$. Foi instituído o tempo de positividade mais rápido em 120 minutos em comparação com a hemocultura periférica, conforme Bouza et al. ${ }^{10} \mathrm{em}$ artigo publicado em 2007, em que comparando-se os diferentes métodos de diagnóstico de ICSRC, concluiu-se que este método tem maior sensibilidade e um maior valor preditivo negativo. Também entrou como critério o início dos sinais clínicos de infecção do cateter 48 horas após entrada do paciente na enfermaria, conforme protocolo descrito de infecção por Marschall et al ${ }^{7}$.

A coleta da hemocultura foi realizada conforme protocolo da instituição, através da coleta de $10 \mathrm{ml}$ de sangue do CVC após descarte dos $10 \mathrm{ml}$ iniciais, e $10 \mathrm{ml}$ de sangue de veia periférico, em tubos separados, imediatamente levados para análise.

Todos os casos de infecção tiveram avaliação final de Infectologista do GAV.

Todos os cateteres foram avaliados pelo grupo, mas coube ao médico assistente do paciente a decisão de retirar ou não o dispositivo, tendo sido retirado todos com hemocultura positiva.

Com relação à análise estatística, inicialmente todas as variáveis foram analisadas descritivamente. Para as variáveis quantitativas esta análise observou os valores mínimos e máximos, o cálculo de médias, os desvios-padrão e a mediana. Para as variáveis qualitativas calcularam-se frequencias absolutas e relativas. Para a comparação das médias de dois grupos foi utilizado o teste $t$ de Student.

Para averiguar a homogeneidade entre as proporções utilizou-se o teste $\chi^{2}$ e o teste exato de Fisher (quando ocorreram frequências esperadas menores de cinco). Para a obtenção de fatores preditivos de óbito foi ajustado o modelo de regressão logística multivariada. Os valores de Sensibilidade e Especificidade foram obtidos através da curva ROC. Os valores de odds ratio foram obtidos através da regressão logística.

O software utilizado para a análise foi o SPSS 15.0 para Windows. O nível de significância utilizado para os testes foi de $5 \%$.

Protocolo e estudo aprovados pelo Comitê de Ética da Irmandade Santa Casa de Misericórdia de São Paulo, protocolo $030 / 10$.

\section{Resultados}

Durante o período estudado, foram analisados $114 \mathrm{ca}-$ teteres de 96 pacientes, com resultados descritos na Tabela 1 , sendo que $36,4 \%$ (35) eram do sexo feminino e $63,5 \%$ (61), do sexo masculino. Os pacientes tinham uma idade média de 59 anos, com média de internação de 18 dias na unidade. Infecção relacionada ao cateter ocorreu em 9,64\% dos casos (11 cateteres).

O tempo médio de uso do cateter foi de 12,3 dias, variando de 1 a 69 dias, não tendo sido usado nenhum protocolo de retirada ou troca, conforme exposto no CDC Guidelines $^{6}$, sendo que 31,3\% dos cateteres permaneceram mais de 15 dias. $\mathrm{Na}$ análise estatística em relação à quantidade de dias de uso e infecção, não foi obtida correlação significante $(\mathrm{p}=0,156)$, embora $19,4 \%$ (7 de 36$)$ dos cateteres inseridos com mais de 14 dias tiveram que ser retirados por infecção.

Em relação ao número de lumens, um caso foi excluído, pois não informava com exatidão o número de lumens, obtendo que 54,8\% (62) eram de duplo lúmen e 45,1\% (51), de mono lúmen. Feita análise comparativa, obteve-se que 4 $(7,84 \%)$ dos 51 cateteres mono lúmen evoluíram com infecção contra sete $(11,9 \%)$ dos 62 duplo lúmen, dessa forma, não apresentando diferença estatística $(\mathrm{p}=0,274)$.

Tabela 1. Comparações entre as características dos cateteres infectados e os não infectados.

\begin{tabular}{|c|c|c|c|}
\hline Características & ICSRS positivo & ICSRC negativo & Total \\
\hline Masculino & $9(24,30 \%)$ & $28(76,70 \%)$ & $37(36,60 \%)$ \\
\hline Feminino & $4(6,35 \%)$ & $60(93,65 \%)$ & $64(63,40 \%)$ \\
\hline Idade & 59 anos $(35-74)$ & 52,9 anos $(19-83)$ & Média de 53,46 anos \\
\hline Tempo de uso ${ }^{\text {a }}<14$ dias & $6(7,20 \%)$ & $77(96,80 \%)$ & $83(69,70 \%)$ \\
\hline Tempo de uso $^{\mathrm{a}} \geq 14$ dias & $7(19,40 \%)$ & $29(80,60 \%)$ & $36(30,30 \%)$ \\
\hline Duplo lúmen ${ }^{\mathrm{b}}$ & $8(11,90 \%)$ & $59(88,00 \%)$ & $67(56,30 \%)$ \\
\hline Mono lúmen ${ }^{b}$ & $5(9,60 \%)$ & $47(90,00 \%)$ & $52(43,70 \%)$ \\
\hline Subcláviac & $10(17,24 \%)$ & $48(82,76 \%)$ & $58(48,70 \%)$ \\
\hline Jugularc & $1(1,79 \%)$ & $55(98,20 \%)$ & $56(47,10 \%)$ \\
\hline Femoral $^{c}$ & $1(25,00 \%)$ & $3(75,00 \%)$ & $4(3,40 \%)$ \\
\hline
\end{tabular}

ICSRS - infecção de corrente sanguínea relacionada a cateter venoso central; ${ }^{2}$ valor $p$ sem significância ( $p=0,18$ ); bvalor $p$ sem significância ( $p=0,246$ ); ${ }^{\mathrm{v}}$ alor $\mathrm{p}=0,008$; o protocolo de 1 dos 13 cateteres com infecção não especificava com exatidão o local de passagem, por isto, este foi excluído na análise entre os locais de punção. 
Os agentes encontrados nas hemoculturas foram, em parte, semelhantes à literatura, com cultura polimicrobiana em 2 casos $(18,1 \%)$ e monomicrobiana nos outros 9 casos $(81,8 \%)$. O germe mais comum foi o Staphilococcus coagulase negativo com 5 hemoculturas positivas, seguido do Pseudomonas aeruginosa com 4 hemoculturas positivas.

Ocorreu passagem na subclávia em 58 cateteres (50,9\%) e na jugular em $56(49,1 \%)$. Feita análise estatística com relação aos sítios de inserção, obtiveram-se 17,2\% de infecção na subclávia e 1,8\% de infecção na jugular (Figura 1), com $\mathrm{p}=0,005$. Calculamos o risco relativo (odds ratio) de infecção na subclávia em relação à jugular, da ordem de 11,2(95\% IC: $1,4-92,8)$ vezes maior em relação àquela $(\mathrm{p}=0,023)$.

\section{Discussão}

A literatura tem sido extensa detalhando infecção em cateteres venosos centrais, mas no entanto, a grande maioria dos estudos foi feita em ambiente de terapia intensiva, com pacientes graves, muitas vezes intubados e de difícil manejo. Climo et al. ${ }^{11}$ publicaram em 2003 que de 2.459 pacientes, $29 \%$ deles tinham acesso venoso profundo; $55 \%$ dos pacientes de UTI e $24 \%$ dos pacientes de enfermaria tinham acesso, porém em números absolutos, o número de acessos em enfermaria era maior que em UTI (506 pacientes contra 212), concluindo a importância do cuidado e preparo na inserção dos cateteres. Nosso estudo teve por objetivo avaliar somente pacientes de enfermaria e os de casos cirúrgicos, sem ter o propósito de compará-los aos pacientes de UTI.

Alguns estudos revelam uma incidência de 1 a 13\% de hemoculturas positivas relacionadas a cateter ${ }^{12-15}$, sendo que em nossa instituição ocorreu em 9,64\% (11 cateteres) dos acessos venosos profundos. Encontra-se, portanto no

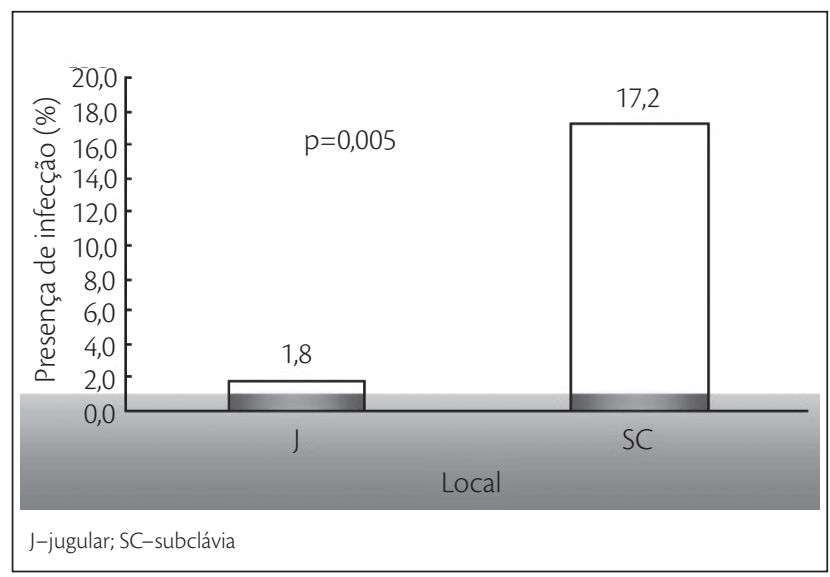

Figura 1. Comparação entre infecção no acesso venoso jugular e no subclávio. limite superior, é mais um número preocupante, mas devese levar em conta que os operadores do nosso serviço geralmente são residentes de primeiro ano, sem uma experiência ideal. Bernard e Stahl ${ }^{13}$ já descreveram que o risco de complicações mecânicas e infecciosas depende da experiência do operador (com número de corte para 50 cateterizações), com uma larga diferença de complicações infecciosas para operadores experientes ( $25 \%$ das complicações) em relação aos não experientes (56\% das complicações) ${ }^{16}$.

Conforme já foi dito, são escassos os trabalhos na literatura especificando infecção de cateter central em ambientes externos a UTI. O primeiro realizado nos EUA, publicado por Marschall em $2007^{7}$, encontrou um índice de infecção comparável com os de UTI, levando a crer que em ambientes externos a esta os cateteres têm maior chance de infectar, provavelmente por serem mais clinicamente injustificáveis e com menos cuidados básicos em relação aos cuidados na UTI.

Com relação ao tempo médio de uso dos cateteres de 12,3 dias, apesar da literatura orientar a retirada destes quando colocados por mais de 14 dias $^{17-19}$, preferiu-se seguir as orientações do CDC Guidelines ${ }^{6}$ para retirar os cateteres somente se a infecção fosse comprovada. Ponto que merece relevância concernente à ampla variação do número de 1 a 69 dias de uso, 30\% dos casos com mais de 14 dias, necessitando de uma revisão urgente e a retirada dos acessos sem necessidade, pois quando ultrapassam esse limite ficam mais suscetíveis à infecção ${ }^{18,19}$. Apesar do fato de 19,4\% dos cateteres com mais de 14 dias terem evoluído com infecção, contra somente $5,1 \%$ dos com menos de 14 dias, não foi obtida correlação estatística.

O local da inserção que oferece menor risco de infecção tem sido ao longo dos tempos palco de controvérsias na literatura, diante de tal quadro, ficou a critério de cada instituição fixar isto em seu protocolo. De acordo com Guideline do CDC publicado em $2002^{6}$, o local preferido de inserção com relação a complicações infecciosas é a subclávia, mas nenhum trial satisfatório foi realizado no intuito de sanar tal dúvida.

Deshpande et al. ${ }^{20}$, em trabalho publicado em 2005, não encontraram diferença estatística com relação à colonização ou infecção de cateter nos três diferentes locais de inserção, em pacientes de UTI. Merrer et al. ${ }^{5}$ publicaram o estudo mais apurado no assunto, demonstrando que cateter na femoral tinha um risco maior em relação à subclávia (19,8\% versus $4,5 \%, \mathrm{p}<0,001)$, mas sem analisar jugular com relação à subclávia. Em estudo publicado em 2005, Lorente et al. ${ }^{21}$ analisaram 2.595 cateteres demonstrando diferença estatística de infecção entre os três locais: o acesso femoral foi associado a uma incidência maior de complicações infecciosas em relação aos outros dois sítios, e jugular esteve associada a uma 
maior incidência significativa de complicações infecciosas que subclávia (risco relativo de 3,1 vezes com $\mathrm{p}=0,005$ ).

$\mathrm{O}$ acesso femoral tem tendência à maior percentagem de infecção, provavelmente isso decorre do fato de neste local ter maior densidade de flora bacteriana na pele. No que tange a jugular em relação à subclávia, ainda especula-se a causa. Provavelmente deve-se a dois fatores: (1) proximidade com a cavidade oral; (2) maior densidade da flora bacteriana devido à maior temperatura local e a dificuldade de manter curativos oclusivos ${ }^{21}$. Mas todos os trabalhos descritos têm o viés de terem sido realizados em ambiente de terapia intensiva, com pacientes com temperatura mais alta, de gravidade maior, alguns traqueostomizados ou entubados, com maior dificuldade de drenar secreções da cavidade oral.

$O$ presente trabalho encontrou uma menor incidência de infecção na jugular em relação à subclávia, diferentemente da literatura descrita, provavelmente porque os pacientes em enfermarias não possuem todas as dificuldades descritas acima. Porém, não se pode afirmar que o acesso jugular proporciona menor chance de infecção, pois necessita de estudos com casuística maior. Os pacientes também não foram randomizados com relação à patologia da internação, apesar de já ter sido publicado na literatura que em pacientes críticos obteve-se menor índice de infecção ${ }^{22}$, levando a crer que existem vários fatores ainda não identificáveis responsáveis por infecção relacionada a cateter.

\section{Conclusão}

Revelamos, com nossos resultados, superioridade do acesso jugular, em relação à presença de infecção, em ambiente fora da UTI, quando comparando com o acesso subclávio.

\section{Agradecimentos}

Agradecemos à Raisa Zoraide Cunha de Melo pelo empenho na revisão gramatical e estruturação contextual.

\section{Referências}

1. Richards M, Edwards J, Culver D, Gaynes R. Nosocomial infections in combined medical-surgical intensive care units in the United States. Infect Control Hosp Epidemiol. 2000;21(8):510-15.

2. Rosenthal V, Guzman S, Pezzotto S, Crnich C. Effect of an infection control program using education and performance feedback on rates of intravascular device-associated bloodstream infections in intensive care units in Argentina. Am J Infect Control. 2003;31(7):405-9.

3. Orsi G, Di Stefano L, Noah N. Hospital-acquired, laboratory-confirmed bloodstream infection: increased hospital stay and direct costs. Infect Control Hosp Epidemiol. 2002;23(4):190-7.
4. Safdar N, Kluger DM, Maki DG. A review of risk factors for catheter-related bloodstream infection caused by percutaneously inserted, noncuffed central venous catheters: implications for preventive strategies. Medicine (Baltimore). 2002;81(6):466-79.

5. Merrer J, De Jonghe B, Golliot F, Lefrant JY, Raffy B, Barre E, et al. Complications of femoral and subclavian venous catheterization in critically ill patients: a randomized controlled trial. JAMA. 2001;286(6):700-7.

6. O'Grady NP, Alexander M, Dellinger EP, Gerberding JL, Heard SO, Maki DG et al. Guidelines for the prevention of intravascular catheter-related infections. Am J Infect Control. 2002;30(8):476-89.

7. Marschall J, Leone C, Jones M, Nihill D, Fraser VJ, Warren DK. Catheterassociated bloodstream infections in general medical patients outside the intensive care unit: a surveillance study. Infect Control Hosp Epidemiol. 2007;28 (8):905-9.

8. Vonberg RP, Behnke M, Geffers C, Sohr D, Ruden H, Dettenkofer M, et al. Device-associated infection rates for non-intensive care unit patients. Infect Control Hosp Epidemiol. 2006;27(4):357-61.

9. Trick WE, Vernon MO, Welbel SF, Wisniewski MF, Jernigan JA, Weinstein RA. Unnecessary use of central venous catheters: the need to look outside the intensive care unit. Infect Control Hosp Epidemiol. 2004;25(3):266-8.

10. Bouza E, Alvarado N, Alcala L, Perez MJ, Rincón C, Muñoz P. A randomized and prospective study of 3 procedures for diagnosis of Catheter-Related bloodstream infection without catheter withdrawal. Clinical Infectious Diseases 2007;44:820-6.

11. Climo M, Diekema D, Warren DK, Herwaldt LA, Perl TM, Peterson L, et al. Prevalence of the use of central venous access devices within and outside of the intensive care unit: results of a survey among hospitals in the prevention epicenter program of the Centers for Disease Control and Prevention. Infect Control Hosp Epidemiol. 2003;24(12):942-5.

12. Goetz AM, Wagener MM, Miller JM, Muder RR: Risk of infection due to central venous catheters: effect of site of placement and catheter type. Infect Control Hosp Epidemiol. 1998,19(11):842-5.

13. Richet H, Hubert B, Nitemberg G, Andremont A, Buu-Hoi A, Ourbak $P$, et al. Prospective multicenter study of vascular-catheter-related complications and risk factors for positive central-catheter culture in intensive care unit patients. J Clin Microbiol. 1990;28(11):2520-5.

14. Moro ML, Vigano EF, Cozzi Lepri A. Risk factors for central venous catheter-related infections in surgical and intensive care units. The Central Venous Catheter Related Infections Study Group. Infect Control Hosp Epidemiol. 1994;15(4 Pt 1):253-64.

15. Sadoyama G, Gontijo Filho PP. Comparison between the jugular and subclavian vein as insertion site for central venous catheters: microbiological aspects and risk factors for colonization and infection. Braz ) Infect Dis. 2003;7(2):142-8.

16. Bernard RW, Stahl WM. Subclavian vein catheterizations: A prospective study. I. Noninfectious complications. Ann Surg. 1971;173(2):184-90.

17. Richet H, Hubert B, Nitemberg G, Andremont A, Buu-Hoi A, Ourbak $P$, et al. Prospective multicenter study of vascular-catheter-related complications and risk factors for positive central-catheter cultures in intensive care unit patients. J Clin Microbiol. 1990; 28(11):2520-25.

18. Norwood S, Wilkins HE 3rd, Vallina VL, Fernandez LG, McLarty JW. The safety of prolonging the use of central venous catheters: a prospective analysis of the effects of using antiseptic-bonded catheters with daily site care. Crit Care Med. 2000;28(5):1376-82. 
19. Corona ML, Peters SG, Narr BJ, Thompson RL. Infections related to central venous catheters. Mayo Clin Proc. 1990;65(7):979-86.

20. Deshpande KS, Hatem C, Ulrich HL, Currie BP, Aldrich TK, BryanBrown CW, Kvetan $\mathrm{V}$. The incidence of infectious complications of central venous catheters at the subclavian, internal jugular, and femoral sites in an intensive care unit population. Crit Care Med. 2005;33(1):1320; discussion 234-5.

21. Lorente L, Henry C, Martin MM, Jimenez A, Mora ML. Central venous catheter-related infection in a prospective and observational study of 2,595 catheters. Crit Care. 2005;9(6):R631-5.

22. Mnatzaganian G, Galai N, Sprung CL, Zitser-Gurevich $Y$, Mandel $M$, Ben-Hur D, et al. Increased risk of bloodstream and urinary infections in intensive care unit (ICU) patients compared with patients fitting ICU admission criteria treated in regular wards. J Hosp Infect. 2005;59(4):331-42.

Correspondência
Gustavo Lopes Gomes Siqueira
Rua Aureliano Coutinho, 77 - apto 93
CEP 01224-020 - São Paulo (SP), Brasil
E-mail: gustavomed@yahoo.com
Contribuições dos autores
Concepção e desenho do estudo: GLGS, WH
Análise e interpretação dos dados: GLGS, WH
Coleta de dados: GLGS, MAN, DMN
Redação do artigo: GLGS, WH, RAC
Revisão crítica do texto: GLGS, WH, RAC
Aprovação final do artigo*: GLGS, WH, DMN, RAC, MAN
Análise estatística: GLGS, RC
Responsabilidade geral pelo estudo: GLGS
Informações sobre financiamento: GLGS

